\title{
CONTROLE NÃO-LINEAR DE FORÇA DE MÚSCULOS ARTIFICIAIS POLIMÉRICOS POR EFEITO CAPACITIVO
}

\author{
Pedro F. C. Blois de Assis* \\ prbloisegmail.com
}

\author{
Marco Antonio Meggiolaro* \\ meggi@puc-rio.br
}

* Depto. de Engenharia Mecânica, Pontifícia Universidade Católica do Rio de Janeiro

Rua Marquês de São Vicente 225, Gávea, Rio de Janeiro, RJ - Brasil 22453-900

\begin{abstract}
Non-Linear Force Control of Polymeric Artificial Muscles Based on Capacitive Effects

An incipient technology for actuation with physical and dynamic characteristics similar to natural muscles has been studied since early 90's. However, the control of such systems is not a trivial task, due to actuator non-linearities. This work presents a proposal for non-linear control of force in artificial muscles based on the VHB4905 acrylic polymer. Mathematical models of the tested actuator are developed. Experiments are performed on a especially developed test bench with a force transducer and the artificial muscle actuated by a high voltage circuit ( $10 \mathrm{kV}$ maximum). A maximum force error of $1 \%$ is obtained when comparing the model that characterizes the material and the experiment that measures force versus electric stimulus. It is shown that the proposed non-linear controller results in a better step response when compared to a standard PID controller. The standard PID does not take into account the system non-linearities, therefore it cannot avoid oscillations in the step response when subjected to high voltages, close to the dielectric breakdown of the polymer. The effectiveness of the proposed control technique is proven experimentally.
\end{abstract}

KEYWORDS: Artificial Muscles, Dielectric Polymers, Force Control.

\footnotetext{
Artigo submetido em 15/09/2009 (Id.: 01033)

Revisado em 07/01/2010, 29/04/2010, 27/05/2010

Aceito sob recomendação do Editor Associado Prof. Marco Henrique Terra
}

\section{RESUMO}

Uma tecnologia incipiente para atuação de sistemas com características físicas e dinâmicas parecidas com músculos naturais vem sendo estudada desde o início da década de 90. No entanto, o controle destes sistemas não é uma tarefa trivial, devido às não-linearidades intrínsecas. Este trabalho apresenta uma proposta para controle não-linear de força em músculos artificiais baseados no polímero acrílico VHB4905. São desenvolvidos modelos matemáticos do atuador polimérico experimentado. Essas equações são utilizadas no controle de forma a compensar os efeitos não-lineares do sistema. Experimentos são conduzidos em uma bancada de testes especialmente desenvolvida, contendo um sensor de força e o atuador polimérico acionado por um circuito de alta tensão (até $10 \mathrm{kV}$ ). Obtém-se um erro máximo de força de $1 \%$ entre o modelo que caracteriza o material e o experimento de força em função do estímulo elétrico. Demonstrase que o controle não-linear proposto gera melhores resultados a entradas em degrau que um controle PID padrão, o qual não leva em consideração a não-linearidade e a alta sensibilidade do atuador quando submetido a tensões próximas da tensão de quebra do dielétrico, gerando oscilações. A eficácia da técnica de controle proposta é comprovada experimentalmente

PALAVRAS-CHAVE: Músculos Artificiais, Polímeros Dielétricos, Controle de Força

\section{INTRODUÇÃO}

O objetivo de muitos engenheiros robóticos tem sido desenvolver robôs autônomos capazes de lidar com missões de alto 
nível. No entanto, o desenvolvimento desses robôs tem sido limitado pela complexidade da tecnologia de atuação, controle e potência, que ainda são incomparáveis aos simples sistemas do mundo natural [Kim, Tadokoro, 2007].

Polímeros ativos com características similares ou melhores que os músculos biológicos são uma grande promessa para o desenvolvimento de sistemas biologicamente inspirados (o biomimetismo). Baixa rigidez e grandes deformações são algumas das características similares ao músculo natural. Eles são mais flexíveis que os motores convencionais e podem agir como amortecedores de impactos e vibrações. Essas características habilitam o desenvolvimento de dispositivos mecânicos sem embreagens, rolamentos ou outros mecanismos responsáveis pelos grandes custos e complexidades [Bar-Cohen, 2003].

Uma área de aplicação onde essa tecnologia seria de suma importância é a prostética. Pessoas acidentadas que perderam um ou ambos os membros superiores enfrentam duas principais conseqüências. A primeira consequência é funcional, a partir do momento em que o amputado fica impossibilitado de exercer tarefas como manipular e segurar objetos. A segunda consequência é psicológica, pois a amputação modifica a aparência da extremidade superior. Apesar dos anos de pesquisas e inovações, as próteses para mão, disponíveis atualmente, não lidam com esses problemas adequadamente.

O trabalho de [Pons et al., 2004] mostra o resultado de dois conjuntos de questionários que foram distribuídos para 200 amputados e 150 profissionais de reabilitação. Cerca de $60 \%$ dos questionários foram respondidos levando às seguintes estatísticas: as principais preocupações dos amputados são estética (62\%), desconforto (58\%), excesso de peso $(58 \%)$, falta de capacidade funcional $(50 \%)$, problemas com assistência técnica e manutenção (31\%), barulho (27\%) e problemas com a parte restante do membro amputado (e.g. irritação e suor) (15\%).

De forma a atender às necessidades dos pacientes, uma prótese de mão deve ter os seguintes requisitos:

Funcionalidade - a prótese deve segurar de maneira estável uma grande variedade de objetos com formas complexas;

Estética - ela deve ter a mesma forma, peso e dimensões da mão humana;

Controlabilidade - ela deve ser fácil de operar.

Para avaliar a sua funcionalidade, é importante notar que próteses de mão geralmente são projetadas para segurar objetos e não para manipulá-los. Este último requer alta destreza, sensores avançados, estratégias de controle avançadas e interfaces naturais para o controle. O projeto de tal prótese, capaz de segurar e manipular objetos, só será possível quando o desenvolvimento de novos componentes de alta tecnologia permitir a realização de estruturas mecânicas bastante compactas e complexas [Carrozza et al, 2004]. Para isso é fundamental que músculos artificiais que acionem estas próteses possuam um adequado controle de força.

Uma tecnologia que propõe suprir as deficiências apontadas anteriormente é a de polímeros ativos, em particular os EAP, polímeros eletroativos. Uma de suas características mais atraentes é o seu potencial de atuação para o biomimetismo, pois são leves, baratos, elásticos, silenciosos, ágeis e necessitam de pouca potência. Essas características são atraentes também para aplicações espaciais. Entre os anos de 1995 e 1999 um estudo feito pela NASA [Bar-Cohen et al., 1999] teve o objetivo de aumentar o entendimento e a viabilidade dos materiais à base de polímeros eletroativos e identificar aplicações planetárias. Os materiais investigados incluem IPMC e polímeros dielétricos, que foram usados como atuadores curvos e longitudinais respectivamente. Os dispositivos que foram desenvolvidos incluem um varredor de sujeira, uma garra, e um braço robótico [Bar-Cohen, 2004]. Para uso em próteses, é necessário que estudos de controle de força sejam feitos nestes polímeros.

Este trabalho tem como objetivo o estudo do controle de força de músculos artificiais poliméricos, com o auxílio de uma bancada de testes com sensor de força. Um controlador não-linear é proposto para melhorar a resposta do sistema a degraus. Na próxima seção, o princípio de atuação destes polímeros é descrito.

\section{MÚSCULOS ARTIFICIAIS - POLÍME- ROS DIELÉTRICOS (DEAP)}

O desenvolvimento de músculos artificiais a partir de polímeros dielétricos é uma área bastante incipiente no mundo e principalmente no Brasil. Em outros países, estudos foram feitos nessa área no que diz respeito à caracterização física e dinâmica do material [Wingert, 2002; Wissler \& Mazza, 2006]. Já existem diversas aplicações para essa tecnologia, incluindo atuadores lineares, sensores, micro-posicionadores etc. O desafio continua sendo encontrar uma forma de utilizar esses atuadores de uma maneira mais prática para que um dia esta tecnologia possa ser usada, e.g., nos principais atuadores de uma prótese de membro superior, ou em manipuladores igualmente fortes aos atuais, com a vantagem de serem mais leves e de consumirem menos energia.

Assim como nos músculos naturais, a força produzida por músculos artificiais varia com o nível de estímulo. Resultados preliminares mostram que o valor máximo de tensão 
obtido por polímeros ativos (Electroactive Polymers, EAP), $800 \times 10^{3} \mathrm{Nm}^{-2}$, situa-se acima da faixa de valores medidos para músculos naturais. A deformação para a qual esses materiais mostram maior potência, 2,5\%, encontra-se próxima ao limite inferior da faixa dos valores medidos para músculos naturais [Bar-Cohen, 2004]. Deve-se levar em consideração que EAPs são capazes de deformações (quando estimulados) maiores que $100 \%$ [Pelrine et al., 2000].

Os atuadores EAP possuem a capacidade tanto de geração quanto de absorção de energia semelhante à dos músculos naturais. Uma potência máxima de $40 \mathrm{Wkg}^{-1}$ foi alcançada em um ciclo de $10 \mathrm{~Hz}$, com deformação de $2,5 \%$ a uma tensão de estímulo de 6kV [Bar-Cohen, 2004]. Esses resultados mostraram que a potência gerada pelo atuador encontrase dentro da fronteira dos valores para os músculos naturais quando próximos da ativação máxima.

Um tipo de atuador polimérico em especial estudado por esse trabalho é o polímero dielétrico (Dielectric Electroactive Polymer - DEAP). Este material consiste numa fita adesiva de dupla face (VHB4905 da 3M) e é capaz de deformar mais de $100 \%$.

Os DEAP são atuados através de campos elétricos de alta intensidade pelo princípio do efeito capacitivo entre eletrodos flexíveis colocados nas superfícies de maior área do material. Esses eletrodos podem ser graxa condutora depositada no polímero com o auxílio de pincéis. Desta maneira, o polímero age como dielétrico entre os eletrodos formados pelas graxas.

Essa fita adesiva (VHB4905 da 3M) possui, dentre outras, as propriedades de baixa rigidez elástica e grande constante dielétrica, permitindo portanto a aplicação de altas tensões elétricas (da ordem de $\mathrm{kV}$ ) entre os eletrodos, sob as quais grandes deformações são observadas envolvendo forças significativas [Pelrine et al., 1998].

Seu funcionamento como atuador pode ser explicado pela força de atração eletrostática (força de Maxwell) entre as placas do capacitor (condutores flexíveis depositados no polímero) gerada através da grande diferença de potencial aplicada nas mesmas. Isso ocorre devido às cargas elétricas opostas que se acumulam nas superfícies do dielétrico. Essa atração entre as superfícies do polímero comprime o mesmo na direção de sua espessura. Como esse material possui um coeficiente de Poisson muito próximo a 0,5 (o volume se conserva), as outras duas dimensões livres (comprimento e largura) expandem, como mostra a Figura 1. Superposta à força de Maxwell, existe a força de repulsão entre cargas elétricas iguais existentes em cada eletrodo, contribuindo para a expansão das dimensões livres [Pelrine et al., 2000].

A pressão efetiva, responsável pela deformação do material durante a estimulação do atuador, pode ser expressa pelo modelo eletrostático
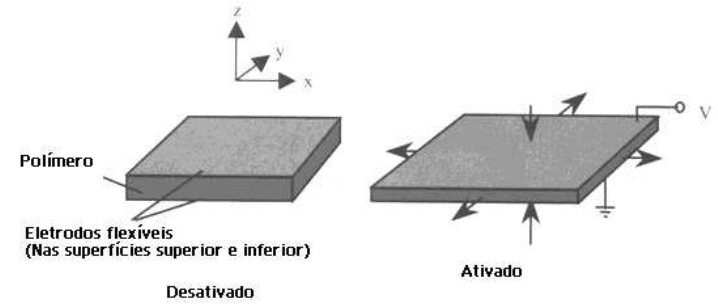

Figura 1: Princípio de operação de atuadores com polímeros dielétricos [Bar-Cohen, 2004].

$$
P=e_{r} e_{0}\left(\frac{V}{z}\right)^{2}
$$

onde a pressão $\mathrm{P}$ é função da tensão elétrica aplicada, $\mathrm{e}_{\mathrm{r}}$ é a permissividade relativa do polímero (constante dielétrica), $\mathrm{e}_{0}$ é a permissividade do vácuo, $\mathrm{V}$ é a tensão elétrica aplicada e z é a espessura do polímero.

O estado final de deformação do atuador varia de acordo com a carga aplicada (estímulo elétrico), as condições de contorno do sistema e do modulo de elasticidade do polímero que varia com a deformação aplicada no mesmo. Estudos feitos mostram que a constante dielétrica do material pode ser maior pré-tensionando (aplicando uma deformação inicial ao atuador mecanicamente, antes do estímulo elétrico) o polímero. Essa constante pode variar de $18 \mathrm{MV} / \mathrm{m}$ sem deformações até $218 \mathrm{MV} / \mathrm{m}$ com uma deformação de $500 \%$ nas duas direções livres (comprimento e largura) [Kofod, 2001]. Com isso, para que possam ser aplicadas maiores tensões elétricas e com isso maiores atuações (tanto deformações quanto esforços), é necessário um pré-tensionamento do polímero. No entanto, essa pré-deformação aplicada mecanicamente ao polímero antes da atuação pode gerar um comportamento anisotrópico do material, tornando o módulo de elasticidade diferente nas direções livres, dificultando bastante a modelagem do polímero para o cálculo da deformação resultante.

\section{MODELAGEM DO ATUADOR}

O atuador considerado neste trabalho consiste do polímero VHB4905 preso a dois pares de barras de fenolite (as barras horizontais na Figura 2). O atuador, antes de ser revestido com graxa condutora, é pré-tensionado na direção x e preso às barras. $\mathrm{O}$ atuador é então pré-tensionado na direção y, ambas as barras são presas a anteparos, e a uma delas é acoplado um sensor de força, representado pela letra F na Figura 2.

O pré-tensionamento gera deformações nas direções x e y 


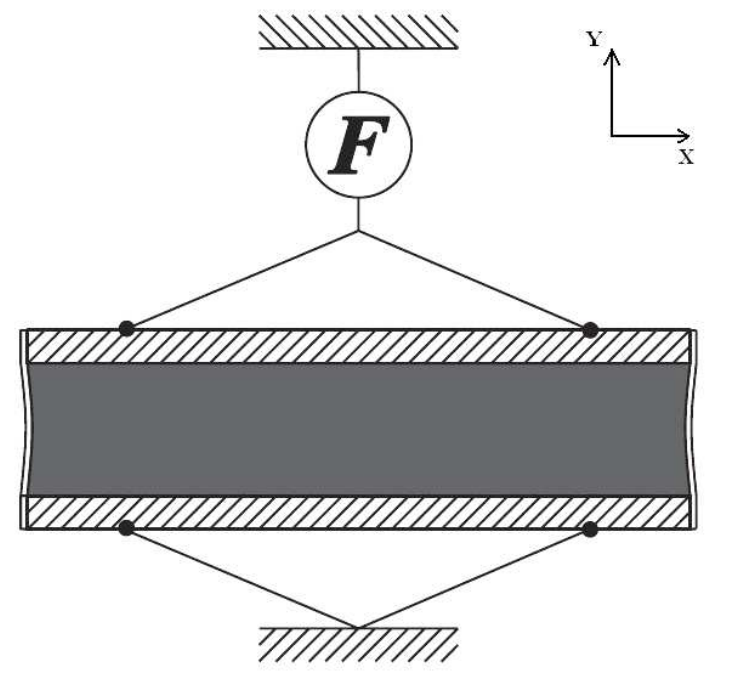

Figura 2: Esboço da montagem do experimento de teste da força do atuador [Kofod, 2001].

definidas por $\varepsilon_{\mathrm{x}, \text { pre }}$ e $\varepsilon_{\mathrm{y}, \text { pre }}$, cujas tensões mecânicas associadas $\sigma_{\mathrm{x}, \text { pre }}$ e $\sigma_{\mathrm{y} \text {,pre }}$ são balanceadas respectivamente pela compressão longitudinal das barras e pelos anteparos (superior e inferior). Desse modo, as tensões, assim como as deformações, em x e y são a soma de duas parcelas, uma relativa ao pré-tensionamento e outra provocada pela tensão elétrica aplicada e pela carga útil $\left(\sigma_{\mathrm{x}}, \sigma_{\mathrm{y}}, \varepsilon_{\mathrm{x}} \mathrm{e} \varepsilon_{\mathrm{y}}\right)$.

As extremidades superior e inferior ao longo da direção $\mathrm{x}$ estão engastadas nas barras, portanto nestas posições não é possível haver deformações adicionais em x, além de $\varepsilon_{\mathrm{x}, \text { pre }}$, e portanto tem-se $\varepsilon_{\mathrm{x}}=0$. Como a dimensão do atuador em y é muito menor do que em $x$, pode-se assumir que a deformação $\varepsilon_{\mathrm{x}}$ em todo o polímero seja pequena, ou mesmo que $\varepsilon_{\mathrm{x}} \cong 0$ para todo o polímero, mesmo em sua região central.

Como ambas as barras não podem se movimentar na direção y, e o polímero foi pré-tensionado mecanicamente em y para evitar flambagem, pode-se concluir também que $\varepsilon_{\mathrm{y}}=0$, e assim

$$
\varepsilon_{x}=\frac{1}{E}\left[\sigma_{x}-v\left(\sigma_{y}+\sigma_{z}\right)\right]=0 \Rightarrow \sigma_{x}=v\left(\sigma_{y}+\sigma_{z}\right)
$$

$$
\varepsilon_{y}=\frac{1}{E}\left[\sigma_{y}-v\left(\sigma_{x}+\sigma_{z}\right)\right]=0 \Rightarrow \sigma_{y}=v\left(\sigma_{x}+\sigma_{z}\right)
$$

onde $v$ é o coeficiente de Poisson e E o Módulo de Young do material. A tensão na direção z, por sua vez, corresponde à componente da pressão responsável pela atração entre os condutores, e vale $\sigma_{\mathrm{z}}=-\mathrm{P} / 2$. Substituindo a Equação (3) na Equação (2), tem-se então

$$
\begin{aligned}
\sigma_{x} & =-\frac{P}{2} \frac{v}{1-v} \\
\sigma_{y} & =-\frac{P}{2} \frac{v}{1-v}
\end{aligned}
$$

Pode-se ver que as tensões mecânicas nas direções x e y são compressivas, como deveriam ser, pois foi assumido que nessas direções não haveria deformações. O polímero está livre para se deformar apenas em z, a direção associada à sua espessura, resultando em

$$
\varepsilon_{z}=\frac{1}{E}\left[\sigma_{z}-v\left(\sigma_{y}+\sigma_{x}\right)\right]=\frac{P}{E}\left[\frac{v^{2}}{1-v}-\frac{1}{2}\right]
$$

Por outro lado, como o comportamento do polímero é nãolinear, na presença de altas deformações o Módulo de Young $E$ nas equações precisa ser substituído pelos valores $E_{x}, E_{y} e$ $\mathrm{E}_{\mathrm{z}}$ medidos nas direções $\mathrm{x}, \mathrm{y}$ e $\mathrm{z}$ após o pré-tensionamento. Desse modo, pré-tensionamentos de intensidades distintas em x e y irão provocar um comportamento anisotrópico do músculo artificial. Ao incluir a anisotropia do material, as Equações (2-6) resultam em

$$
\begin{gathered}
\varepsilon_{x}=\frac{\sigma_{x}}{E_{x}}-v\left(\frac{\sigma_{y}}{E_{y}}+\frac{\sigma_{z}}{E_{z}}\right)=0 \\
\varepsilon_{y}=\frac{\sigma_{y}}{E_{y}}-v\left(\frac{\sigma_{x}}{E_{x}}+\frac{\sigma_{z}}{E_{z}}\right)=0 \\
\sigma_{x}=-\frac{P}{2} \frac{v}{1-v} \frac{E_{x}}{E_{z}} \\
\sigma_{y}=-\frac{P}{2} \frac{v}{1-v} \frac{E_{y}}{E_{z}} \\
\varepsilon_{z}=\frac{\sigma_{z}}{E_{z}}-v\left(\frac{\sigma_{x}}{E_{x}}+\frac{\sigma_{y}}{E_{y}}\right)=\frac{P}{E_{z}}\left[\frac{v^{2}}{1-v}-\frac{1}{2}\right]
\end{gathered}
$$

Note que o valor de $\varepsilon_{\mathrm{z}}$ no caso anisotrópico não depende de $\mathrm{E}_{\mathrm{x}}$ nem $\mathrm{E}_{\mathrm{y}}$. Como não há pré-tensionamento na direção $\mathrm{z}$ (direção da espessura), pode-se assumir que $\mathrm{E}_{\mathrm{z}}$ é igual a $\mathrm{E}$, o Módulo de Young para baixas deformações.

Se for considerado o coeficiente de Poisson como 0,5, de acordo com as Equações (6) e (9) tem-se $\varepsilon_{z}=0$, como seria esperado. Isto porque quando $v=0,5 \mathrm{o}$ material é incompressível, e assim deformações nulas em x e y implicam em $\varepsilon_{\mathrm{z}}=0$ para não violar a conservação de volume.

No entanto, mesmo os elastômeros possuem $v$ um pouco abaixo de 0,5 , resultando em deformações em $\mathrm{z}$ pequenas mas não nulas. Experimentos conduzidos com o elastômero estudado resultaram em um valor calibrado de 0,495 para o coeficiente de Poisson.

\section{CONTROLE DE FORÇA}

Para controlar a força exercida pelo atuador, foi implementado um controlador digital padrão PID com ganhos $\mathrm{K}_{\mathrm{p}}=$ 
$0,005, \mathrm{~K}_{\mathrm{i}}=0,00015$ e $\mathrm{K}_{\mathrm{d}}=0,020$ de forma a obter-se uma resposta estável, com rápida convergência e overshoot máximo de $1 \%$. A Fig. 3 mostra a malha de controle representativa do sistema.

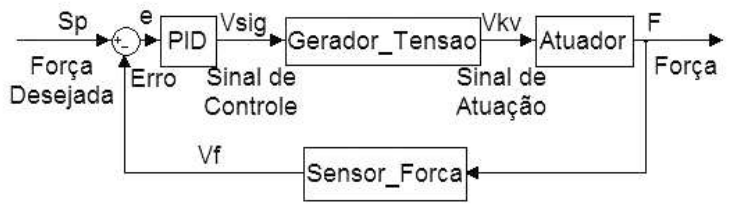

Figura 3: Diagrama de blocos do sistema de controle de força do atuador polimérico.

Desse diagrama pode-se estabelecer uma relação entre a força desejada (representada por uma tensão de referência $\mathrm{S}_{\mathrm{p}}$, medida em $\mathrm{V}$, proporcional à força desejada) e a força de saída do sistema $(\mathrm{F}$, medida em $\mathrm{N}$ ) depois de lida pelo transdutor e convertida no sinal de tensão $\mathrm{V}_{\mathrm{f}}$, medida em $\mathrm{V}$ :

$$
\begin{gathered}
e=S_{p}-V_{f} \\
V_{s i g}=K_{p}\left(e+K_{d} \dot{e}+\int e / K_{i}\right) \\
V_{k V}=V_{s i g} C_{10}+C_{11} \\
F=A P=\frac{x_{0} z e_{r} e_{0} V_{k V}^{2}}{z^{2}}=\frac{x_{0} e_{r} e_{0} V_{k V}^{2}}{z} \\
V_{f}=C_{2} F
\end{gathered}
$$

A Equação (10) mostra o sinal de erro $e$ da malha de controle onde são comparados o valor desejado de força $S_{p}$ e a força atual lida $\mathrm{V}_{\mathrm{f}}$. Esse sinal de erro é então avaliado pelo controlador PID de acordo com a Equação (11), onde $\mathrm{K}_{\mathrm{p}}$ é o ganho proporcional, $\mathrm{K}_{\mathrm{d}}$ é o ganho derivativo, $\mathrm{K}_{\mathrm{i}}$ é o ganho integral, e $\mathrm{V}_{\text {sig }}$ é o sinal de saída do controlador. A Equação (12) mostra o modelo adotado para o gerador de alta tensão, onde o sinal de saída $\mathrm{V}_{\mathrm{kv}}$ é uma função linear da entrada $\mathrm{V}_{\text {sig }}$ com taxa de proporcionalidade $\mathrm{C}_{10}$ e termo independente $\mathrm{C}_{11}$. Estes parâmetros foram estimados através de um ajuste experimental e dependem não só da incerteza do amplificador de tensão usado no atuador, mas também das incertezas dos resistores, amplificadores operacionais e outros componentes utilizados na construção do circuito de alta tensão. A força F exercida pelo atuador é modelada de acordo com a Equação (13), pelo produto da área $\mathrm{A}$ (onde os eletrodos foram aplicados) pela pressão efetiva $\mathrm{P}$ aplicada, onde $\mathrm{x}_{0} \mathrm{e} \mathrm{z}$ são respectivamente a largura e a espessura do atuador, $\mathrm{e}_{\mathrm{r}}$ é a permissividade relativa do polímero, e e é a permissividade do vácuo. Finalmente, a Equação (14) mostra a relação linear entre a força $F$ do atuador e o sinal de tensão $V_{f}$ através da constante $\mathrm{C}_{2}$.

Com essas equações, chega-se à força de saída do sistema quando controlada por um PID linear

$$
F=\frac{A}{z}\left(K_{p}\left(e+K_{d} \frac{d}{d t} e+\frac{1}{K_{i}} \int e \cdot d t\right) C_{10}+C_{11}\right)^{2}
$$

onde $e=\mathrm{S}_{\mathrm{p}}-\mathrm{C}_{2} \cdot \mathrm{F}, \mathrm{A}=\mathrm{x}_{0} \cdot \mathrm{e}_{\mathrm{r}} \cdot \mathrm{e}_{0}, \mathrm{C}_{10} \cong 1071, \mathrm{C}_{10} \cong 210 \mathrm{~V}$, $\mathrm{C}_{2}=3 \times 10^{-3} \mathrm{~V} / 22,24 \mathrm{~N} \pm 0,03 \% \mathrm{FS}$. As Equações (13) e (15) mostram as não-linearidades do sistema, que podem prejudicar o desempenho do controlador. Analisando a Equação (13), nota-se que a força exercida pelo atuador é proporcional não só ao quadrado da tensão elétrica aplicada, mas também ao inverso da espessura do polímero. Isso torna o sistema mais sensível a erros nas tensões próximas à tensão elétrica limite do atuador (tensão de quebra do dielétrico), em comparação ao comportamento do mesmo quando submetido a baixas tensões. O ajuste dos ganhos de um controlador PID linear para otimizar a resposta a degraus de baixas tensões elétricas resulta, em geral, em respostas oscilatórias ou até instáveis para degraus de tensões mais altas.

Note que o atuador retangular duplamente engastado exerce maior força sobre o sensor para baixas tensões elétricas aplicadas, pois o polímero se encontra pré-tensionado na bancada de teste. Por outro lado, quanto maior a tensão aplicada no polímero, maior é a sua tendência de expandir e consequentemente menor será a força medida pelo sensor. Portanto, o comportamento oscilatório observado no PID linear é mais pronunciado nas menores forças, associadas a tensões elétricas mais altas.

Para compensar as não-linearidades descritas, é proposto ajustar os ganhos do controlador de acordo com o estado (F, z) do sistema. A malha de controle resultante é mostrada na Figura 4.

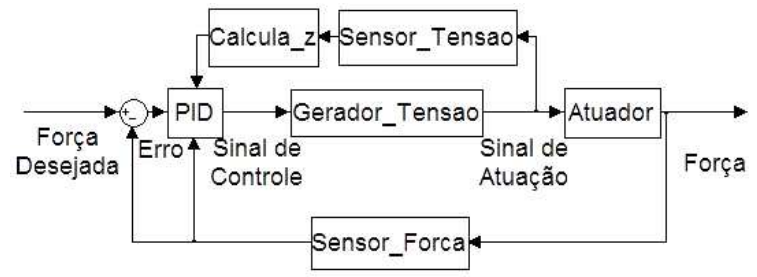

Figura 4: Malha de controle do sistema com a utilização de um controlador PID com ganhos variáveis.

O ajuste proposto consiste em multiplicar o ganho proporcional do controlador (e consequentemente a tensão de atuação 
do polímero) por um fator de ajuste de $(\mathrm{z} / \mathrm{F})^{1 / 2}$, onde a força F é medida pela célula de carga, e a espessura $\mathrm{z}$ é encontrada através do modelo matemático desenvolvido. Desta forma, obtém-se uma relação linear entre a força exercida pelo polímero e a sua tensão de atuação:

$$
\begin{aligned}
& F=\frac{x_{0} e_{r} e_{0}\left(V_{k V} \sqrt{z / F}\right)^{2}}{z}=\frac{x_{0} e_{r} e_{0} V_{k V}^{2}}{z} \frac{z}{F} \\
& \Rightarrow F^{2}=x_{0} e_{r} e_{0} V_{k V}^{2} F=V_{k V} \sqrt{x_{0} e_{r} e_{0}} .
\end{aligned}
$$

Note que o sistema (amplificador) gerador de alta tensão fornece uma saída do tipo $\mathrm{V}_{\mathrm{kV}}=\mathrm{C}_{10} \cdot \mathrm{V}_{\mathrm{sig}}+\mathrm{C}_{11}$. Multiplicandose o ganho proporcional do controlador pelo fator de ajuste mencionado acima, multiplica-se apenas a primeira parcela desta equação. Elevando-se essa tensão à segunda potência, tem-se não só a soma de três termos, mas também um termo com dependência da raiz quadrada da razão da espessura pela força,

$$
\begin{aligned}
& V_{k V}=C_{10} K_{p} e_{q} \sqrt{\frac{z}{F}}+C_{11} \Rightarrow \\
& \Rightarrow V_{k V}^{2}=\left(C_{10} K_{p} e_{q}\right)^{2} \frac{z}{F}+C_{11}^{2}+2 C_{11} C_{10} K_{p} e_{q} \sqrt{\frac{z}{F}}
\end{aligned}
$$

onde: $e_{q}=\left(e+K_{d} \dot{e}+\int e / K_{i}\right)$ Desta forma, a linearização do sistema, proposta acima, não ocorrerá. A solução encontrada para isso é substituir a saída do controlador de uma forma que a alta tensão sobre o músculo artificial seja o produto do sinal de entrada da eletrônica geradora de alta tensão por uma constante, sem a soma de nenhuma outra parcela

$$
\begin{aligned}
& V_{N s i g}=\frac{\left(V_{s i g}-C_{11 / 1000)}\right.}{C_{10 / 1000}} \Rightarrow V_{k V}=C_{10} V_{N s i g}+C_{11} \Rightarrow \\
& \Rightarrow V_{k V}=\frac{\left(V_{\text {sig }}-C_{11 / 1000)}\right.}{C_{10 / 1000}} C_{10}+C_{11}=1000 V_{s i g}
\end{aligned}
$$

Substituindo a Equação (18) na Equação (16), obtém-se a equação do sistema linearizado, dada por

$$
F=1000\left[K_{p}\left(e+K_{d} \frac{d}{d t} e+\int \frac{e}{K_{i}} d t\right)\right] \sqrt{x_{0} e_{r} e_{0}}
$$

Na próxima seção, o programa de controle e sistema elétrico para acionamento dos polímeros são descritos.

\section{SISTEMA EXPERIMENTAL}

\subsection{Sistema Mecânico}

O polímero utilizado é a fita acrílica dupla face VHB4905, produzida pela $3 \mathrm{M}$. A composição química não é informada pelo fabricante, e o material está disponível sob encomenda na forma de um rolo com $0,5 \mathrm{~mm}$ de espessura, e largura e comprimento especificáveis. Todos os testes foram realizados no Laboratório de Robótica da PUC-Rio a uma temperatura aproximada de $23^{\circ} \mathrm{C}$.

Para os testes são utilizadas 4 placas de fenolite de $170 \mathrm{~mm}$ de comprimento, $37 \mathrm{~mm}$ de largura e $4,5 \mathrm{~mm}$ de espessura. $\mathrm{O}$ polímero é esticado em um equipamento desenvolvido no laboratório com perfis de alumínio e preso entre um par dessas molduras (placas).

Suportes usados em bancadas ópticas foram utilizados para fixar o atuador estudado. Estes suportes oferecem ajustes de distâncias com incrementos de décimos de milímetro tanto em altura (suporte-elevador) quanto em comprimento (suporte-separador), e foram montados em uma base de fixação composta de uma placa de alumínio.

Com estes equipamentos, monta-se uma bancada de testes (Figura 5) onde se instala o sensor de força LCEB-5 da Omega Engineering Inc. no suporte-elevador, e uma conexão para pinos no suporte-separador, para fixar diferentes atuadores poliméricos.

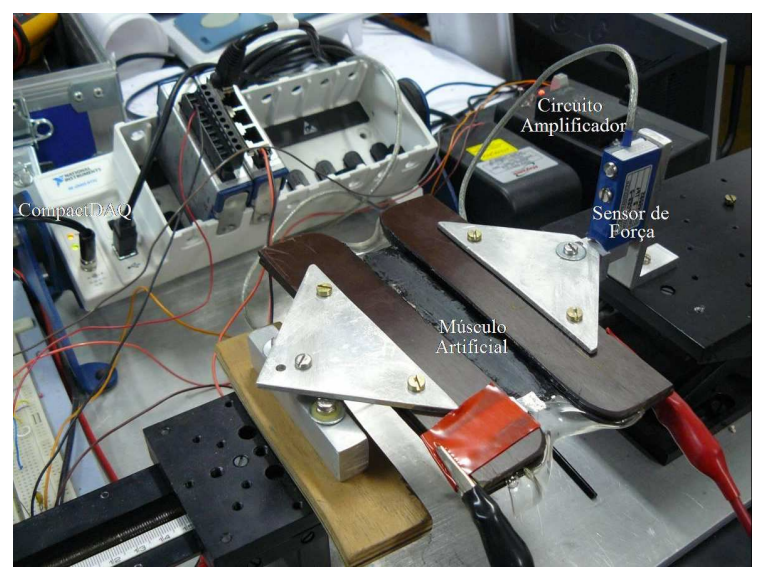

Figura 5: Bancada experimental com o atuador montado.

Sobre o restante da base colocam-se os outros equipamentos de leitura de sinais e atuação do músculo. Estes últimos consistem no circuito de atuação desenvolvido, um módulo CompactDAQ (National Instruments) com entradas e saídas analógicas conectado a um computador, e uma fonte de corrente contínua de $18 \mathrm{~V}$. 
Com a configuração de atuador retangular descrita na Seção 3 (atuador retangular duplamente engastado, Figura 2), diferentes perfis de tensão são aplicados ao polímero. Experimentaram-se perfis como rampas, degraus, senóides e dentes de serra, com diferentes frequências, assim como tensões constantes. Diferentes pré-deformações são também impostas na direção y através da movimentação do suporteseparador.

Não só a força é monitorada, mas também a tensão elétrica aplicada, através de um sensor para altas tensões. Todas as informações são gravadas automaticamente em planilhas. São feitos também testes para obter curvas de força em função da deformação para diferentes valores de tensão. Para tal, varia-se a deformação (de engenharia) do polímero de $100 \%$ a $400 \%$ a uma taxa de $1 \mathrm{~mm}$ a cada 3 minutos, para valores de tensão que variam entre $1,5 \mathrm{kV}$ a $5,5 \mathrm{kV}$.

\subsection{Sistema Elétrico de Acionamento}

O princípio de funcionamento do atuador polimérico estudado envolve altas tensões, da ordem de $10 \mathrm{kV}$, necessárias para que as forças eletrostáticas entre os condutores sejam suficientes para comprimir o polímero e produzir as deformações desejadas. O consumo de corrente do atuador é muito baixo, da ordem de $10 \mu \mathrm{A}$ [Bar-Cohen, 2004]. Com isso, a potência necessária para excitar o atuador também é baixa, da ordem de $100 \mathrm{~mW}$, e pode ser obtida a partir de uma bateria comum de $9 \mathrm{~V}$. Um circuito oscilador seguido por um transformador com uma razão de 1000:1 e um retificador seriam suficientes para gerar essas altas tensões.

No entanto, componentes eletrônicos como capacitores e diodos utilizados nos retificadores que tolerem altas tensões não são facilmente obtidos. As poucas empresas que fornecem componentes para esses níveis de tensão são fornecedores de componentes para alta potência, que toleram não só tensões da ordem de $\mathrm{kV}$ mas também correntes da ordem de $\mathrm{kA}$. Isso faz com que os componentes sejam extremamente grandes e caros.

A solução encontrada foi adquirir um equipamento específico que convertesse uma tensão de corrente contínua (DC) baixa (de 0 a $15 \mathrm{~V}$ ) para as altas tensões necessárias para o projeto (de 0 a $10 \mathrm{kV}$, também DC). O equipamento escolhido foi o conversor DC-DC da Emco High Voltage Corporation modelo E101, que recebe uma tensão de 0 a $15 \mathrm{~V}$ na entrada, drenando uma corrente de até 300mA (dependendo da carga utilizada no lado da alta tensão), e fornece uma tensão de saída proporcional à entrada linear a partir de $0,7 \mathrm{~V}$. Sua tensão de saída chega a até $10 \mathrm{kV}$ com uma corrente máxima de $0,2 \mathrm{~mA}$. Esse equipamento fornece uma saída com uma oscilação de até $1,5 \%$ (pico-a-pico) com uma frequência de cerca de $5 \mathrm{kHz}$.
O ideal seria que a tensão de saída fosse constante, assim como a entrada. No entanto, como o tempo de resposta do atuador é relativamente alto (da ordem de $0,1 \mathrm{~s}$ ) se comparado com a frequência do equipamento, essas oscilações de alta frequência não interferem na movimentação do músculo.

De forma a operar o atuador através do computador, foi preciso desenvolver um circuito que compatibilizasse a potência da saída do micro-computador PC com a da entrada do conversor DC-DC. Como os sistemas de aquisição típicos fornecem uma tensão de saída de baixa potência (de 0 a $10 \mathrm{~V}$ com no máximo $1 \mathrm{~mA}$ de corrente), desenvolveu-se um circuito capaz de fornecer a potência necessária ao conversor de alta tensão.

Para testes rápidos, que não necessitem do computador para fornecer formas de onda controladas, o circuito desenvolvido também possui um módulo que regula as altas tensões através de um potenciômetro de ajuste. Dessa forma, o atuador pode ser ativado utilizando-se ou o computador ou um potenciômetro, escolhidos através de uma chave seletora de dois estados.

O circuito foi então desenvolvido da seguinte maneira: os sinais de referência do computador e do potenciômetro são ligados nas duas primeiras portas de um multiplexador analógico, e a sua saída é conectada a um buffer de potência. Este último, por sua vez, é composto de um amplificador operacional e de um transistor. Como as entradas são tensões de referência que variam de 0 a $10 \mathrm{~V}$ e o conversor DC-DC recebe uma tensão de 0 a $15 \mathrm{~V}$, o amplificador operacional foi ajustado de forma a ter um ganho de 1,5.

Para que o usuário saiba qual das entradas está selecionando no multiplexador, foram colocados também dois LED's de cores diferentes que são acesos alternadamente de acordo com a chave seletora (vermelho para o computador e verde para o potenciômetro). A lógica de acionamento dos LED's foi feita utilizando-se transistores no modo de chaves comutadoras.

\subsection{Software de Controle}

Foi desenvolvido um programa computacional utilizando LabVIEW8.0 para fazer os testes no sistema experimental descrito acima, que inclui atuação sobre o sistema, leitura dos dados, e a gravação dos mesmos em arquivos do MSExcel. Nele, o usuário pode ver gráficos da tensão aplicada no músculo, da força produzida, e da tensão de entrada no sistema eletrônico desenvolvido, vide Figura 6.

Valores médios da alta tensão e da força do músculo também são disponibilizados. Os perfis de tensão possíveis são senóide, triangular, quadrado, dente de serra e constante, onde 


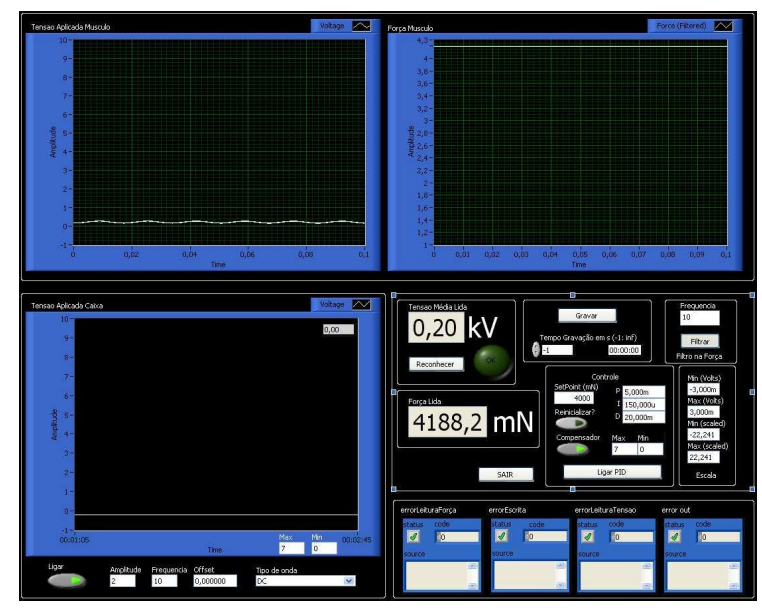

Figura 6: Tela do software de controle da bancada de teste desenvolvida.

as amplitudes, frequências e offsets são configuráveis. Para segurança, o sistema possui um botão para ligar a alta tensão, um algoritmo que detecta a quebra do dielétrico e desliga a atuação de modo a preservar o módulo de alta tensão E101, e limites para tensões máximas e mínimas nos atuadores. Um botão para iniciar e terminar a gravação de dados pode ser visto em cima do mostrador do tempo de gravação corrente. Há ainda o módulo do controlador do sistema com possibilidade de ativar ou não a compensação das não-linearidades do sistema nos ganhos do PID. Por último, é possível alterar a frequência de corte do filtro digital implementado para o sinal de força capturado, bem como as constantes para a conversão dos valores lidos do sensor para valores de força em $\mathrm{N}$.

Os resultados experimentais obtidos com o sistema desenvolvido são apresentados a seguir.

\section{RESULTADOS EXPERIMENTAIS}

\subsection{Experimentos em Malha Aberta}

Os testes em malha aberta têm o objetivo de verificar o modelo matemático desenvolvido da força do atuador em função da tensão elétrica aplicada. Os testes são feitos com um atuador construído com uma camada dupla de polímero VHB4905, pré-deformado de 400\% na direção x (deformação de engenharia) e com uma deformação (também de engenharia) de $360 \%$ na direção y da Figura 2. A secção transversal do polímero considerada para o cálculo das tensões foi calculada como o produto da largura da placa de fenolite (utilizada para prender o polímero) pela largura do polímero calculada a partir da modelagem feita. Para verificar o modelo desenvolvido, é aplicada uma tensão com perfil de rampa crescente até a quebra do dielétrico (aproximadamente
$7,5 \mathrm{kV}$ depois de $2 \mathrm{~min}$ de teste). A Figura 7 mostra a comparação direta entre a curva experimental e a teórica, obtidas pelas Equações (2-6).

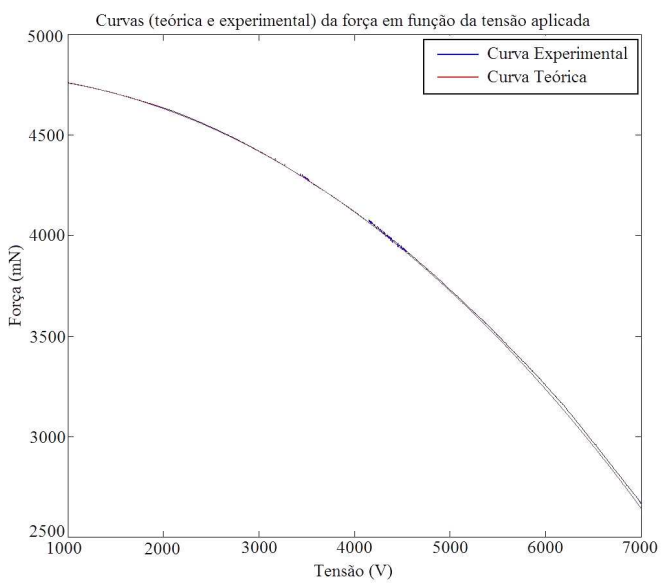

Figura 7: Comparação entre as curvas experimental e teórica da variação da força em função da tensão aplicada no músculo artificial.

O erro médio quadrático do resultado obtido é de $10,5 \mathrm{mN}$. O erro máximo do modelo desenvolvido é de $26,7 \mathrm{mN}$, cerca de $1 \%$ do valor experimental. Esse erro máximo ocorre para uma tensão de aproximadamente $7 \mathrm{kV}$. Isso se deve ao fato de o modelo desenvolvido não contemplar a viscoelasticidade do material. Com isso, o efeito de relaxamento do polímero, sobreposto à atuação, se torna significativo ao final do experimento. Provavelmente, se a taxa de incremento da atuação fosse mais lenta, esse erro final seria maior.

Outros experimentos são conduzidos para diferentes valores de pré-deformação na direção y, aplicado pela movimentação do suporte-separador. São testadas deformações de engenharia em y entre 100 e $400 \%$. As Figuras 8 e 9 mostram as curvas de tensão mecânica pela deformação para tensões elétricas variando de $1,5 \mathrm{kV}$ até $5,5 \mathrm{kV}$. Em cada figura existem 9 curvas, cada uma gerada com um estímulo elétrico diferente. Cada curva corresponde a um estímulo elétrico de $500 \mathrm{~V}$ a mais que a curva logo acima, sendo que a curva com os maiores valores de tensão mecânica corresponde ao menor estímulo elétrico aplicado $(1,5 \mathrm{kV})$.

Nota-se, como esperado, que quanto maior o estímulo elétrico aplicado, menor a tensão mecânica gerada pelo atuador. No entanto, as curvas não se encontram igualmente espaçadas, apesar de o estímulo elétrico entre uma curva e a imediatamente superior ser o mesmo da curva imediatamente inferior.

O mesmo pode ser visto ao longo do eixo da deformação. Quanto maior a deformação, maior a distância entre as cur- 


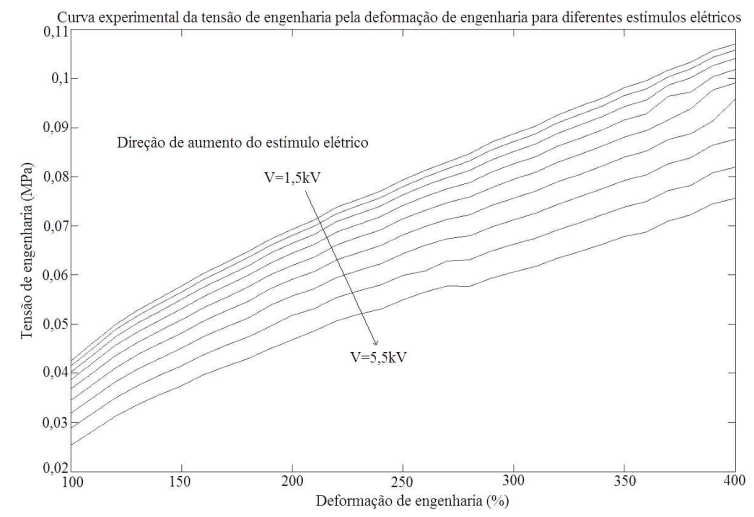

Figura 8: Curvas de tensão mecânica de engenharia pela deformação de engenharia para diferentes estímulos elétricos.

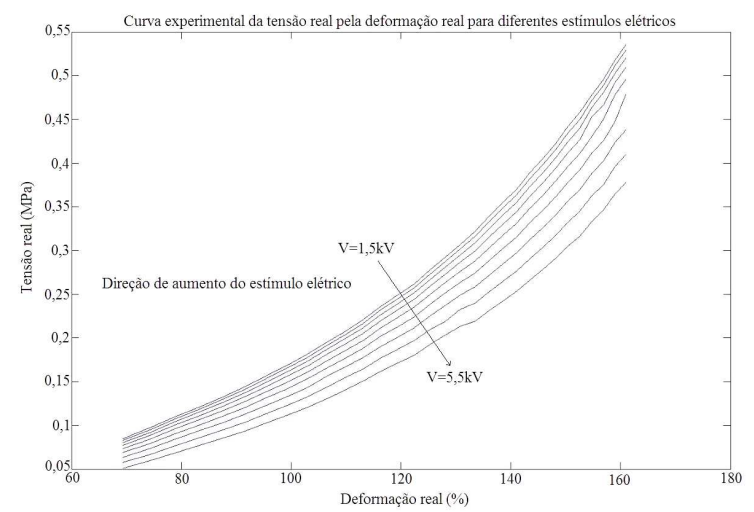

Figura 9: Curvas de tensão mecânica real pela deformação real para diferentes estímulos elétricos.

vas, apesar de o incremento no comprimento do atuador ter sido constante durante o experimento. Esse comportamento se deve às não linearidades apontadas anteriormente, pois a força do polímero varia proporcionalmente ao quadrado do estímulo elétrico aplicado e é inversamente proporcional à espessura do material. Logo, quanto maior a deformação do atuador no seu comprimento, menor a sua espessura e com isso maior a força produzida.

\subsection{Experimentos com o Controlador Proposto}

Experimenta-se aqui o atuador desenvolvido usando um controlador de força PID padrão (linear), onde os ganhos são constantes, e comparam-se os resultados com os do controle não-linear proposto. Por não levar em conta as nãolinearidades do sistema, o PID padrão pode prejudicar a estabilidade do atuador, pois com os ganhos constantes o controlador não é capaz de ajustar a saída de um sistema que possui uma dinâmica significativamente diferente dependendo da tensão elétrica aplicada e da sua espessura.

Na realização dos testes, são aplicados diversos degraus de valores desejados de força em diferentes faixas de atuação do sistema, de modo a explorar os efeitos não-lineares detalhados anteriormente.

Os ganhos de um PID linear são ajustados de forma a encontrar uma resposta estável e rápida para a malha fechada de controle de força, baseados nas medições em tempo real do sensor de força utilizado. Nota-se que os ajustes feitos para baixos valores desejados de força não são satisfatórios para altos valores desejados. O sistema passa a ter um aumento considerável na amplitude das oscilações e um aumento também na sua freqüência. Esse comportamento está de acordo com o modelo desenvolvido da malha de controle com o PID padrão. As não-linearidades do sistema o tornam muito sensível para atuações próximas ao estado de quebra do dielétrico. Um pequeno degrau na atuação tem respostas muito diferentes se o atuador encontrar-se perto da sua tensão limite ou longe da mesma.

Para compensar estes problemas, testa-se também o controlador não linear proposto, descrito anteriormente. Desta maneira, o controlador é ajustado automaticamente ao sistema, fornecendo um comportamento dinâmico similar para qualquer estado em que o polímero se encontre.

O controle não-linear ajusta seus ganhos automaticamente de acordo com a espessura e força de atuação do polímero. Para tal, inicialmente os ganhos de um PID tradicional são ajustados para otimizar a resposta medida de um degrau específico. Em seguida, programa-se o ajuste automático desses ganhos a partir dos modelos não-lineares do sistema, e gravam-se as respostas do sistema a degraus de diversas amplitudes.

Para que possam ser feitas comparações justas e diretas entre os controladores, utiliza-se no PID tradicional o mesmo ganho proporcional do controle não-linear $\left(K_{p} \sqrt{z / F}\right)$ adquirido para altos valores desejados de força). Com isso esperase que o comportamento dos dois controladores seja muito parecido para os valores desejados de força nos quais foram calibrados, pois ambos terão ganhos proporcionais, derivativos e integrais muito próximos.

A principal diferença entre os comportamentos (mostrados nas Figuras 10 e 11) é a regularidade da atuação do controle não-linear para todos os valores desejados de força, com o mesmo comportamento suave e tempos de ascensão e estabilização similares. O PID padrão, apesar de possuir um bom comportamento para valores desejados próximos a $4000 \mathrm{mN}$, para valores menores gera comportamento muito oscilatório e com overshoot significativo. Já o controle não linear não apresenta estes problemas. 


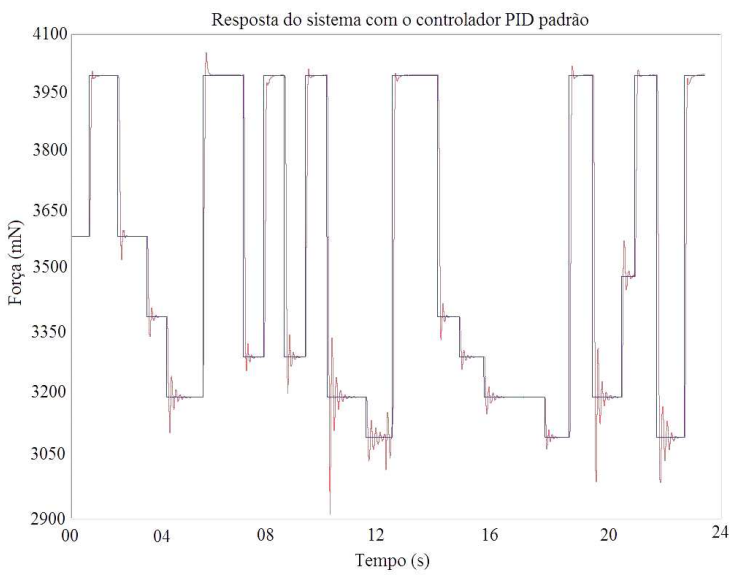

Figura 10: Resposta a diversos degraus do sistema controlado pelo PID padrão, medida experimentalmente, com ganhos ajustados para otimizar a resposta ao degrau de $4000 \mathrm{mN}$. Note o comportamento oscilatório para degraus mais baixos

Outro fator importante é que devido às grandes oscilações do PID padrão, não é possível alcançar valores desejados abaixo de $3100 \mathrm{mN}$, pois as oscilações na tensão de atuação atingem $6 \mathrm{kV}$, próximo do limite de ruptura do polímero. É preciso lembrar que as maiores forças geradas pelo atuador correspondem às menores tensões elétricas aplicadas, como explicado anteriormente.

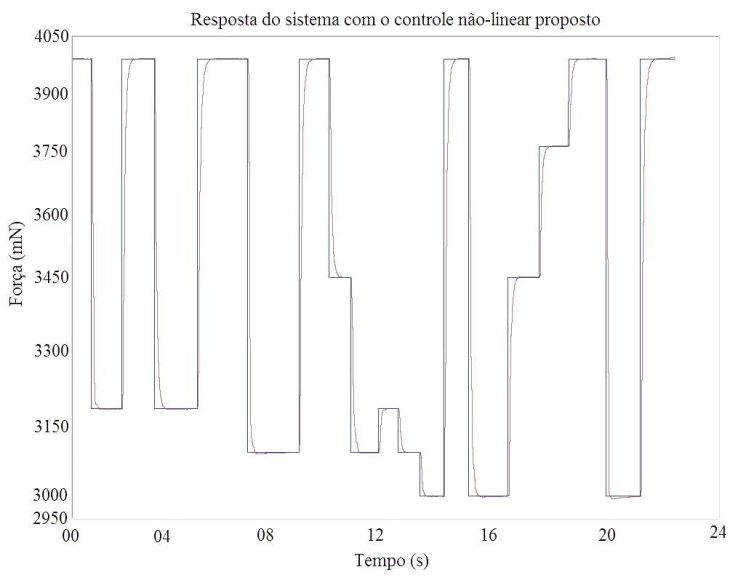

Figura 11: Resposta a diversos degraus do sistema com o controle não-linear proposto.

Nas duas figuras seguintes (Figuras 12 e 13) são mostrados os perfis de tensão aplicados experimentalmente no atuador durante os controles PID padrão e não-linear, respectivamente. Nelas podem-se ver os efeitos da viscoelasticidade do material através da tensão de controle. No controle não-linear, percebe-se na Figura 13 que depois de atingido um baixo valor desejado de força (correspondente a altas tensões elétricas aplicadas vistas na figura), a tensão elétrica comandada decai um pouco. Isso se deve ao relaxamento viscoelástico do material depois de uma mudança nas tensões mecânicas internas. Este relaxamento tenderia a deixar a força aplicada abaixo do valor desejado, o que é compensado automaticamente pelo controle pela diminuição da tensão elétrica, elevando assim a força até os níveis desejados.

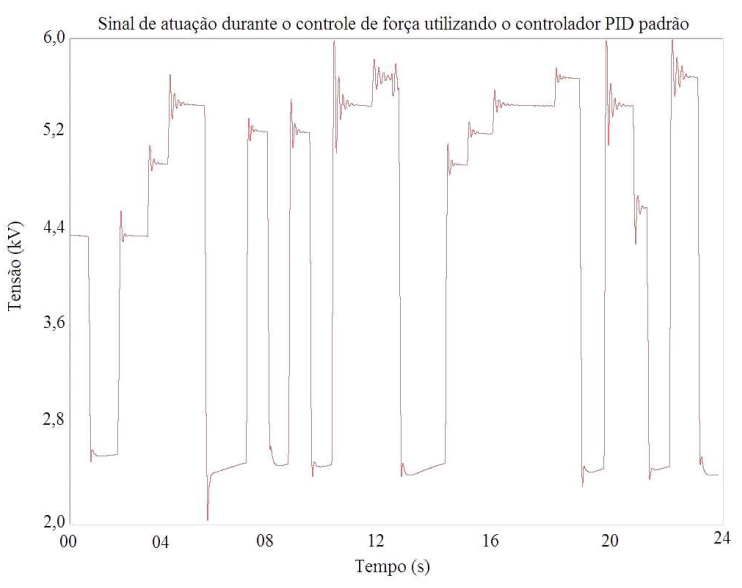

Figura 12: Tensões elétricas aplicadas no atuador durante o controle de força utilizando PID padrão.

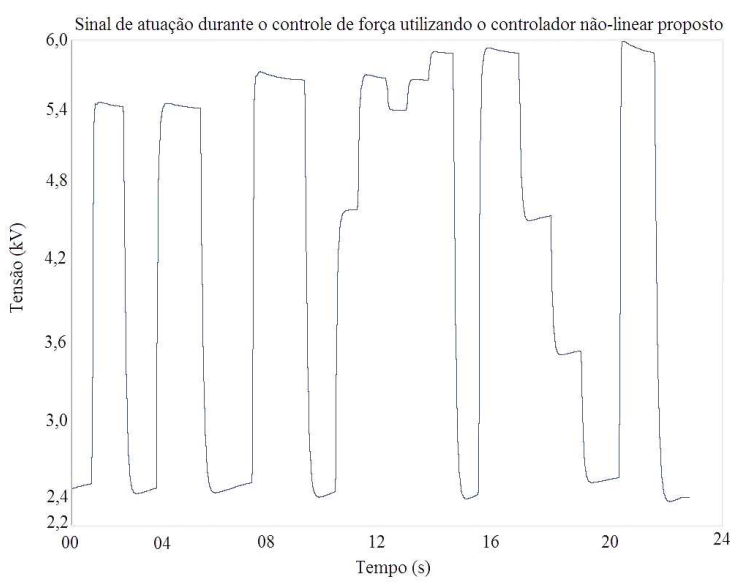

Figura 13: Tensões elétricas aplicadas ao atuador durante o controle de força utilizando a metodologia não-linear proposta. Note a suave variação de tensão exigida para manter o polímero em cada patamar de força, compensando-se assim os efeitos viscoelásticos.

\section{CONCLUSÃO}

Neste trabalho, foi estudado o comportamento de atuadores à base de polímeros eletroativos dielétricos. Testes de força em músculos artificiais baseados no polímero acrílico VHB4905 comprovaram as suas não-linearidades inerentes, identificadas durante a modelagem matemática do sistema. Estas não- 
linearidades causam excessiva sensibilidade do atuador em altas tensões elétricas. Foram programados dois controladores, um PID linear padrão e um controle com um modelo preditivo para compensação das não-linearidades do material. Os resultados mostraram que no PID tradicional a resposta a degrau do sistema, calibrada para um certo nível de força desejada, torna-se oscilatória para menores níveis desejados, associados a maiores tensões elétricas. No PID linear, quanto menor as forças desejadas, maiores as oscilações tanto em amplitude quanto em frequência para a resposta a degrau. $\mathrm{O}$ controlador não-linear proposto corrige estes problemas, apresentando resposta a degrau similar para altos ou baixos valores desejados, sem a necessidade de recalibragem dos ganhos.

\section{REFERÊNCIAS BIBLIOGRÁFICAS}

Bar-Cohen, Y., "Actuation of biologically inspired intelligent robotics using artificial muscles", Industrial Robot: An International Journal, 30(4):331-337, 2003.

Bar-Cohen Y.,Electroactive polymer (EAP) actuators as artificial muscles: reality, potential, and challenges, 2nd ed., SPIE, Washington, 2004.

Bar-Cohen Y., Leary, S., Yavrouian, A., Oguro, K., Tadokoro, S., Harrison, J., Smith, J., "Challenges to the transition of IPMC artificial muscle actuators to practical application", Proceedings of the Fall MRS Symposium: Electroactive Polymers, Boston, 1999.

Carrozza, M. C., Suppo, C., Sebastiani, F., Massa, B., Vecchi, F., Lazzarini, R., Cutkosky, M. R., Dario, P., "The SPRING Hand: Development of a Self-Adaptive Prosthesis for Restoring Natural Grasping", Autonomous Robots (16): 125-141, 2004.

Kim, K. J., Tadokoro, S., Electroactive Polymers for Robotics Applications: Artificial Muscles and Sensors, Springer, New York, USA, 2007.

Kofod, G., "Dielectric elastomers actuators", Ph.D. Thesis, Department of Chemistry, Technical University of Denmark, 2001.

Pelrine R., Kornbluh R., and Joseph J. P., "Electrostriction of polymer dielectrics with compliant electrodes as a mean of actuation", Sensor Actual. A, Vol. 64, pp. 7585, 1998.

Pelrine R., Kornbluh R., Pei Q. and Joseph J. P., "HighSpeed electrically actuated elastomers with strain greater than 100\%",Science 287, pp. 836-839, 2000.

Pons, J. L., Rocon, E., Ceres, R., Reynaerts, D., Saro, B., Levin, S., Van Moorleghem, W., "The MANUS-HAND
Dextrous Robotics Upper Limb Prosthesis: Mechanical and Manipulation Aspects", Autonomous Robots (16): 143-163, 2004.

Wingert A. R., "Development of a Polymer-Actuated Binary Manipulator", Submitted to the Department of Mechanical Engineering in Partial Fulfillment of the Requirements for the Degree of Master of Science in Mechanical Engineering at the Massachusetts Institute of Technology, June, 2002.

Wissler, M., Mazza, E., "Mechanical behavior of an acrylic elastomer used in "Dielectric elastomer actuator", Sensors and Actuators A 134 (2007) 494-504, June, 2006. 